\title{
Development of a High Spectral Resolution Lidar for day-time measurements of aerosol extinction.
}

\author{
Alessia Sannino ${ }^{1}$, Antonella Boselli ${ }^{2}$, Domenico Maisto ${ }^{3}$, Alberto Porzio ${ }^{4}$, Changbo Song ${ }^{5}$, Nicola Spinelli $^{1}$, Xuan Wang $^{4 *}$ \\ ${ }^{1}$ Consorzio Nazionale Interuniversitario per le Scienze Fisiche della Materia (CNISM) \\ and Dip. Fisica "E. Pancini”, Università degli Studi di Napoli Federico II, Napoli, Italy. \\ ${ }^{2}$ Istituto di Metodologie per l'Analisi Ambientale (IMAA) - CNR, Italy. \\ ${ }^{3}$ Consorzio Nazionale Interuniversitario per le Scienze Fisiche della Materia (CNISM) and Dip. Fisica, Politecnico di \\ Milano, Italy. \\ ${ }^{4}$ Istituto Superconduttori, Materiali innovativi e Dispositivi (SPIN) - CNR, Italy. \\ ${ }^{5}$ Beijing Research Institute of Telemetry (BRIT), Beijing, PRC.
}

\begin{abstract}
Lidar technique is the most performing way to obtain the atmospheric vertical profile of aerosol optical properties with high space-time resolution. With elastic scattering lidars, the retrieval of aerosol optical properties (as the extinction profile) is realizable only with assumptions on aerosol extinction-to-backscatter ratio or with Raman measurement achievable in night-time. In order to overcome these problems, the High Spectral Resolution Lidar (HSRL) technique has been examined. In this paper we present an innovative prototype of High Spectral Resolution Lidar realized at Physics Department of University "Federico II" of Naples for the LISA (LIdar for Space study of the Atmosphere) project in the framework of the China-Italy international cooperation between CNISM and BRIT. The prototype which represents a first step of a spaceborne HSRL, is based on a laser source at $1064 \mathrm{~nm}$ and $532 \mathrm{~nm}$ with high spectral resolution ability at $532 \mathrm{~nm}$. The separation between the molecular and the aerosol components was obtained through the use of a confocal Fabry-Perot interferometer (CFPI) cavity.
\end{abstract}

\section{Introduction}

Aerosol particles are among the main constituents of the atmosphere. They are the principal responsible for the uncertainties in the knowledge of the atmospheric processes and of the Earth radiation balance. In fact, their presence and involved direct and indirect processes play a crucial role in the dynamics of the climate change and in the human health and safety.

Due to their crucial role a good knowledge on their characteristics and classification is necessary. In order to obtain range resolved information about the optical properties of the aerosol particles, Lidar (Light Detection and Ranging) remote sensing technique has been proved to be the performing way. The principal optical particles properties collected by lidar are related to concentration, chemical-physical and geometrical particles properties. In particular, lidar systems measurements allow to detect the attenuated backscattering signal; the main particles information derivable from this signal are the backscattering coefficient $(\beta)$ and the extinction one $(\alpha)$. Different types of lidar have been developed based on different physical processes. The most common type is the elastic lidar, based on the elastic scattering of light by atmospheric particles. This lidar type allows to collect direct information on the $\beta$ profiles, while for $\alpha$ retrieval a strong a-priori assumption on the backscattering to extinction ratio must be done [1].

The Lidar based on the Raman molecular process overcome this limit but, due to the lowest cross section of the processes compared to the elastic one, Raman lidar can be used only in night time [2].

A third lidar type which overcomes both previously cited limits is the High Spectral Resolution Lidar (HSRL).

HSRL as the Raman one, returns a direct knowledge on the $\alpha$ and $\beta$ coefficient but without limitations to night time measurements. This can be reached by using the different Doppler broadening of scattering between aerosol particles (Mie scattering) and Rayleigh scattering from molecules. In fact, the molecules undergo a Doppler shift of $\sim 1 \mathrm{GHz}$ due to their thermal motion. Whereas for the aerosol a Doppler shift of about $\sim 30 \mathrm{MHz}$ is expected due to wind. [3] This difference allows to distinguish two distributions coming from the aerosols and molecules contributions. [4]

In the framework of the China-Italy international cooperation project LISA (LIdar for Space study of the Atmosphere) an HSRL has been designed, assembled and tested at department of Physics "Ettore Pancini" of University Federico II - Napoli. [5]

\section{HSRL LISA}

LISA project started with the intent to develop a spaceborne HSRL. In this work the realization of a ground based prototype as a first step of the project will be discussed. The HSRL prototype is equipped with a Cassegrain telescope of $250 \mathrm{~mm}$ diameter and $200 \mu \mathrm{rad}$ field of view. The laser is a pulsed Nd:YAG, operating at

\footnotetext{
Corresponding author: xuan.wang@spin.cnr.it
} 
the fundamental $1064 \mathrm{~nm}$ and $532 \mathrm{~nm}$ wavelengths. The high spectral resolution ability has been applied at 532nm. The spectral analysis system is arranged, after the telescope, by a dichroic mirror, to separate the light in two beams; the IR beam is detected by a Si-APD while the HSRL technique is applied to the return for the $532 \mathrm{~nm}$ wavelength signal.

Before going to HSRL channel, the $532 \mathrm{~nm}$ backscatter signal is split into the parallel and cross polarized channels in order to achieve additional information on aerosols shape. A first stage of the spectral analysis of the signal is performed by the use of an interferential filter and a planar Fabry Perot interferometer. The parallel polarized component is then analysed by a Confocal Fabry Perot Interferometer (CFPI) filter [6]. The aerosol part is transmitted by the cavity while the molecular part is reflected. A frequency locking system is used to tune the receiver frequency to the laser source in order to ensure cavity stability at resonance frequency. [5]

The optical scheme of LISA prototype is reported in Fig.1 and its principal system characteristics are discussed below.

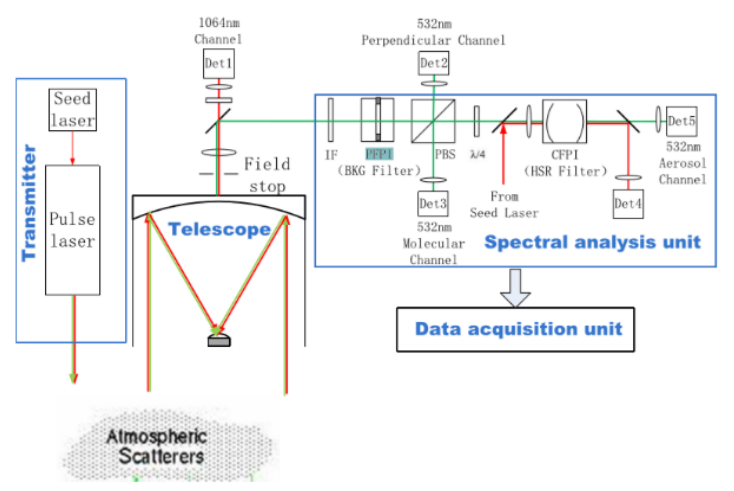

Fig. 1. Operating LISA prototype system.

\subsection{Laser}

The laser has been especially designed and realized for LISA by Bright Solutions Srl in order to have high stability in space application. A $1064 \mathrm{~nm} 1 \mathrm{kHz}$ pulse seed laser has been used and its band width is about $50 \mathrm{MHz}$. The $1 \mathrm{kHz}$ pulsed seed is converted to $20 \mathrm{~Hz}$ repetition rate and amplified to $200 \mathrm{~mJ}$. The second harmonic generator produce about $100 \mathrm{~mJ} 532 \mathrm{~nm}$ laser beam and this wavelength is used for HSRL detection.

\subsection{Confocal Fabry-Perot interferometer (CFPI)}

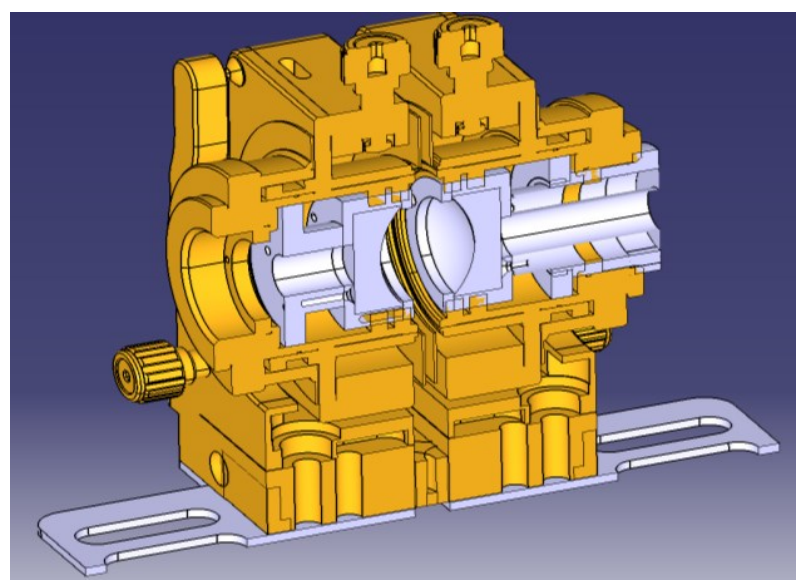

Fig. 2. CFPI scheme. The incoming total light is divided in the two component: aerosols is transmitted after the transition in the cavity; the molecular part is back reflected by mirrors cavity

The confocal Fabry-Perot interferometer (CFPI) is the narrow-band optical filter which has been selected in order to separate the two components of the backscattering light. It transmits the selected wavelength from aerosol scattering and reflects the molecular contribution.

The choice to separate the two spectral contributions by means of CFPI method, instead of more common atomic and molecular absorption filters [5], allows to overcome problems related to the spectral stability of the laser source. On the other hand, the CFPI require more specific expedients. In fact, to enable the CFPI to reflect the molecular part and transmit the aerosol's one, the distance between the two CFPI mirrors needs be accurately controlled. Moreover, the cavity is kept tuned to the fundamental frequency of the laser source. In this condition it acts as a very narrow spectral filter even at the frequency of the second harmonic. This realization of the frequency locking system required the study of cavity dispersion phenomena at two wavelengths. For this HSRL a solid bar piezo actuator (Etalon) moved by a software has been selected. The software for frequency locking has been developed in the project frame.

The main uncertainties in the retrieval of the two signal components can be primarily attributed to three sources: noise from the detector, errors due to deviation in the CFPI transmission, and errors in retrieval of the molecular and aerosol signals due to fluctuation in the CFPI transmission.

These sources have been estimated and the results are shown in the next tables.

In Tab. 1 the first error type related to the detector noise is reported, where $\beta$ is the backscattering coefficient, LR is the extinction to backscattering ratio, Stot_a and Stot_m are the numbers of received photo-electrons for aerosol and molecular signals respectively. The horizontal and vertical resolution are $50 \mathrm{~km}$ and $1 \mathrm{~km}$ respectively.

\begin{tabular}{|c|c|c|c|}
\hline $\begin{array}{c}\text { Aerosol } \\
\text { conditions }\end{array}$ & $\begin{array}{c}\text { Aerosol } \\
\text { Parameters }\end{array}$ & $\begin{array}{c}\text { Signals } \\
\text { (\# Photons) }\end{array}$ & $\begin{array}{c}\text { Relative signals } \\
\text { error }\end{array}$ \\
\hline
\end{tabular}




\begin{tabular}{|c|c|c|c|}
\hline $\begin{array}{c}\text { Low } \\
\text { pollution }\end{array}$ & $\begin{array}{l}\beta=2 \mathrm{E}-6 \\
\mathrm{LR}=50\end{array}$ & $\begin{array}{c}S_{\text {tot } a}=6816 \\
S_{\text {tot } m}=12899\end{array}$ & $\mathrm{e} 1_{a}=2.1 \%$ \\
$\mathrm{e} 1_{m}=2.2 \%$
\end{tabular}

Tab.1 The relative error of the retrieved molecular and aerosol signals at ground due to the detector noise.

The relative errors of the retrieved molecular and aerosol signals at the ground altitude caused by the deviation of the CFPI transmission are reported in Tab. 2

\begin{tabular}{|c|c|c|}
\hline $\begin{array}{c}\text { Aerosol } \\
\text { conditions }\end{array}$ & $\begin{array}{c}\text { Aerosol } \\
\text { parameters }\end{array}$ & $\begin{array}{c}\text { Error due to } \\
\text { CFPI } \\
\text { Transmission }\end{array}$ \\
\hline Low & $\beta=2 \mathrm{E}-6$ & $\Delta \mathrm{e}_{a}=0.08 \%$ \\
pollution & $\mathrm{LR}=50$ & $\Delta \mathrm{e}_{m}=0.09 \%$ \\
\hline Medium & $\beta=1 \mathrm{E}-5$ & $\Delta \mathrm{e}_{a}=0.04 \%$ \\
pollution & $\mathrm{LR}=50$ & $\Delta \mathrm{e}_{m}=0.24 \%$ \\
\hline $\begin{array}{c}\text { High } \\
\text { pollution }\end{array}$ & $\beta=2 \mathrm{E}-5$ & $\Delta \mathrm{e}_{a}=0.04 \%$ \\
$\mathrm{LR}=50$ & $\Delta \mathrm{e}_{m}=0.44 \%$ \\
\hline
\end{tabular}

Tab.2 The relative error of the retrieved molecular and aerosol signals at ground caused by the deviation of the CFPI transmission.

At last the evaluation of the relative errors of the retrieved molecular and aerosol signals at the ground altitude introduced in the retrieval of CFPI transmission are reported in Tab.3

\begin{tabular}{|c|c|c|}
\hline $\begin{array}{c}\text { Aerosol } \\
\text { conditions }\end{array}$ & $\begin{array}{c}\text { Relative error } \\
\text { on } \\
\text { molecular signal }\end{array}$ & $\begin{array}{c}\text { Relative } \\
\text { Error on aerosol } \\
\text { signal }\end{array}$ \\
\hline Low & $\mathrm{e}_{a}=2.1 \%$ & $\mathrm{e} 2_{a}=0.87 \%$ \\
pollution & $\mathrm{e}_{m}=2.2 \%$ & $\mathrm{e} 2_{m}=1.1 \%$ \\
\hline Medium & $\mathrm{e}_{a}=1.1 \%$ & $\mathrm{e} 2_{a}=0.58 \%$ \\
pollution & $\mathrm{e}_{m}=4.9 \%$ & $\mathrm{e} 2_{m}=3.7 \%$ \\
\hline High & $\mathrm{e}_{a}=1.0 \%$ & $\mathrm{e} 2_{a}=0.6 \%$ \\
pollution & $\mathrm{e}_{m}=8.8 \%$ & $\mathrm{e} 2_{m}=7.6 \%$ \\
\hline
\end{tabular}

Tab.3 The relative errors of the retrieved molecular and aerosol signals at the ground altitude caused by the fluctuation of the CFPI transmission.

The relative errors of the retrieved molecular and aerosol signals due to the fluctuation of the CFPI transmission are larger than the errors due to the deviation of the CFPI transmission. However, they are also lower than the errors due to the detector noise.

All these considerations on the errors are derived by simulations and the results obtained meet the design requirements. First measures for the experimental determination of the system's capacities are currently underway.

\section{Conclusion}

This work shows a new High-Resolution Lidar System (HSRL) for spaceborne application, designed, assembled and tested at Physics Department of Università "Federico II" di Napoli. The system is based on the combined use of an interference filter, a planar Fabry-Perot interferometer (PFPI) and a confocal Fabry-Perot interferometer (CFPI). A frequency-locking subsystem has been used to lock the central transmission wavelength to the wavelength of the emitted laser.

LISA spaceborne HSRL will allows three kinds of measurement results. Firstly, the aerosol backscatter coefficients and extinction coefficients can be directly retrieved without any pre-assumption. Secondly, the aerosol linear depolarization ratio at $532 \mathrm{~nm}$ wavelength is retrieved by parallel and cross polarized detection channels. Thirdly the colour index parameter, i.e. the wavelength dependence of the backscattering coefficient, will be retrieved by combining $1064 \mathrm{~nm}$ and $532 \mathrm{~nm}$ channels to obtain quantitative information on the particles size. These retrieved parameters could be rearranged to obtain aerosol concentrations and distinguish aerosol types.

\section{References}

1. Klett, J. D., Stable analytic inversion solution for processing lidar returns, Appl. Opt., 20, 211-220, 1981.

2. Albert Ansmann, Ulla Wandinger, Maren Riebesell, Claus Weitkamp, and Walfried Michaelis, "Independent measurement of extinction and backscatter profiles in cirrus clouds by using a combined Raman elastic-backscatter lidar," Appl. Opt. 31, 7113-7131 (1992)

3. C. Weitkamp, "Lidar Range-Resolved Optical Remote Sensing of the Atmosphere", Singapore: Springer; 2005

4. X. Wang, C. Song, A. Boselli, M. Iarlori, V. Rizi, N. Spinelli, "System simulation of spaceborne and airborne high spectral resolution lidar for aerosol monitoring", XXXVIII Meeting of the Italian Section of the Combustion Institute, doi: 10.4405/38proci2015.XI3.

5. C. Song, "Development of the Spectral Analysis System for a Spaceborne High Spectral Resolution Lidar", PhD Thesis Università Federico II di Napoli, 2014-2017

6. Hoffman D.S., "Confocal Fabry-Perot Interferometer Based High Spectral Resolution Lidar", PhD Thesis, Montana State University, Bozeman, 3 (2012). 